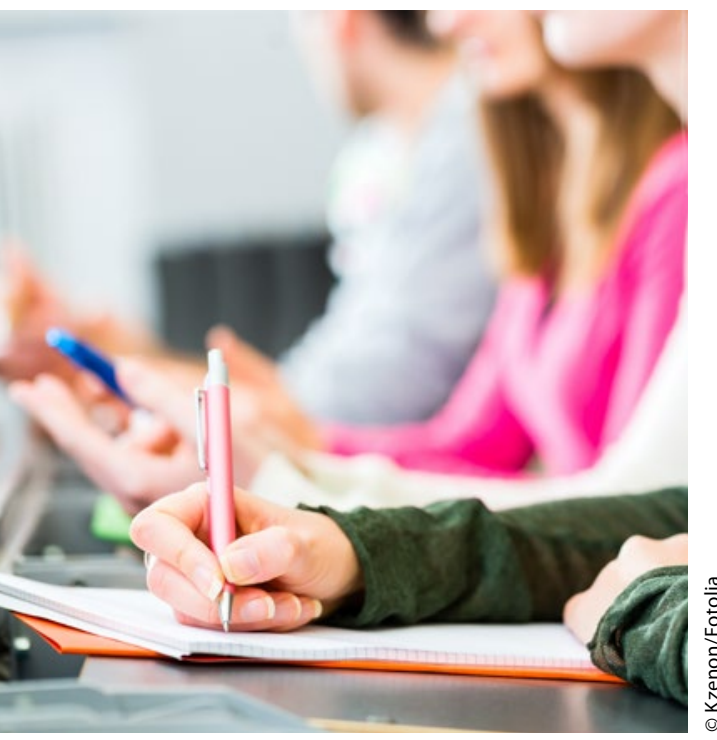

Neuer Studiengang

\title{
Gerontologie und Geriatrie - In Kontakt
}

\begin{abstract}
— Am ersten April 2016 geht am Studienzentrum für Gesundheitswissenschaften \& Management der Dualen Hochschule Baden-Württemberg Stuttgart der berufsbegleitende Kontaktstudiengang "Gerontologie \& Geriatrie" an den Start. Es sollen Pflegende angesprochen werden, die sich beruflich weiterentwickeln und sich ein differenziertes Wissen für die Versorgung geriatrischer und gerontopsychiatrischen Patienten aneignen möchten.

Das Modul wurde gemeinsam mit dem Bundesverband Geriatrie e.V. entwickelt und ist von diesem als Teil der Zercur Geriatrie Fachweiterbildung Pflege zertifiziert. Mit einer Dauer von sechs Monaten ist der berufsbegleitende Kontaktstudiengang in kompakten Präsenzterminen von zwei bis vier Tagen im Monat organisiert. Abgeschlossen wird der Studiengang mit einem Zertifikat und zehn ECTS-Punkten (Leistungspunkten). Darüber hinaus werden mit dem Abschluss die abrechnungsrelevanten Anforderungen für den Arbeitgeber entsprechend der OPS Regelungen erfüllt. Die Teilnahme am Kontaktstudiengang ist gebührenfrei und auch für Pflegende ohne Hochschulberechtigung möglich.
\end{abstract}

www.dhbw-stuttgart.de/pflegewissenschaften

\section{Studium in Heidelberg \\ Interprofessionelle Qualifizierung}

— Schon seit dem Wintersemester 2011/12 bietet die Medizinische Fakultät der Universität Heidelberg den Bachelorstudiengang "Interprofessionelle Gesundheitsversorgung B.Sc." an. Neben der Vermittlung grundlegender Kenntnisse wissenschaftlichen Arbeitens fördert der Studiengang, die Zusammenarbeit zwischen den medizinischen Gesundheitsberufen, um die patientenzentrierte Versorgung zu unterstützen. Für Pflegekräfte mit Berufserfahrung besteht sogar die Möglichkeit des Quereinstieges in das sechste Semester. Das Studium kann in Voll- oder Teilzeit absolviert werden
- beispielsweise mit einer Teilzeitstelle als Kranken- und Gesundheitspfleger. Dadurch kann die Flexibilität und Vereinbarkeit mit dem Beruf zusätzlich erhöht werden. Der erfolgreiche Abschluss des Studiums befähigt zur Berufstätigkeit in der interprofessionellen Gesundheitsversorgung in Gesundheitszentren, im Qualitäts- und/oder Projektmanagement sowie in der Forschung und die weitere Qualifizierung durch andere Masterstudiengänge bis hin zur Promotion.

www.klinikum.uni-heidelberg.de

\section{Weiterbildungsangebot \\ Mehr Qualität in der Versorgung von Diabetikern}

— Pünktlich zum neuen Jahr 2016 startet die Weiterbildung Diabetes-Pflegefachkraft DDG in sieben Bundesländern. 2001 haben bpa und IIGM mit der Fortbildung zur Diabetes-Pflegefachkraft ein Leuchtturmprojekt begonnen. Ziel war es, die Versorgungssituation von Pflegebedürftigen mit Diabetes in ambulanter pflegerischer Betreuung und Pflegeheimen zu verbessern. Seitdem wurde die Fortbildung in enger Zusammenarbeit mit der Arbeitsgemeinschaft Diabetes und Geriatrie der Deutschen Diabetes Gesellschaft (DDG) kontinuierlich weiterentwickelt und für den Bedarf der Pflege optimiert. Mit der DDG und dem bpa ziehen erstmalig eine ärztliche Fachgesellschaft und ein Pflegeverband in der Diabetologie als wesentliche Partner an einem Strang.

www.iigm.de

\section{Kongress \\ Integrative Health \& Medicine}

— Im Sommer gibt es in Deutschland eine große Premiere für die Integrative Medizin: In der Stuttgarter Messe findet vom 9.-11. Juni 2016 erstmalig der „International Congress for Integrative Health \& Medicine" statt. Damit entsteht ein neuer, global und interdisziplinär ausgerichteter Fachkongress, um Expert/innen aus Praxis, Forschung und Politik zusammenzubringen.

Der Kongress ist als Brückenschlag zwischen Praxis und Forschung konzipiert und richtet sich an alle Gesundheitsberufe, die an der Integrativen Medizin interessiert sind. Im interprofessionellen Dialog zwischen Ärzten, Pflegenden und Therapeuten geht es darum, sinnvolle Lösungen für die drängenden Probleme der globalen Gesundheitssysteme zu finden. Das Programm ist so aufgebaut, dass internationale Expert/innen anhand von aktuellen Forschungsergebnissen, Best-Practice-Modellen und Konzepten zeigen, wie konkrete Therapievorschläge für chronische Krankheitsbilder wie Diabetes, Bluthochdruck, Krebs oder Zudem werden Perspektiven für eine moderne Gesundheitsversorgung vorgestellt, die eng mit den Gesundheitszielen der Weltgesundheitsorganisation (WHO) verknüpft sind.

www.icihm.org 